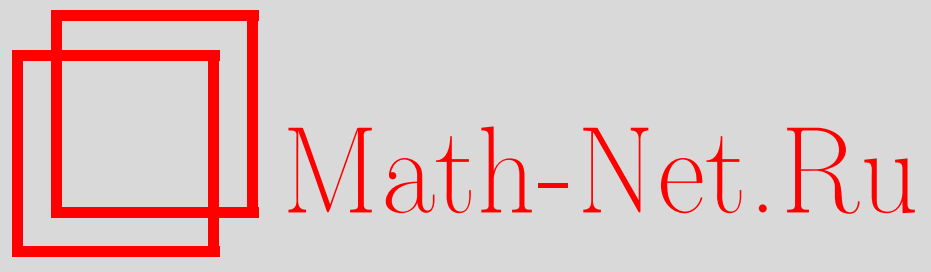

Р. И. Ямилов, Условия интегрируемости для аналогов релятивистской цепочки Тоды, ТМФ, 2007, том 151, номер 1, 66-80

DOI: https://doi.org/10.4213/tmf6012

Использование Общероссийского математического портала Math-Net.Ru подразумевает, что вы прочитали и согласны с пользовательским соглашением http://www . mathnet.ru/rus/agreement

Параметры загрузки:

IP : 52.87 .193 .239

26 апреля 2023 г., 18:26:17

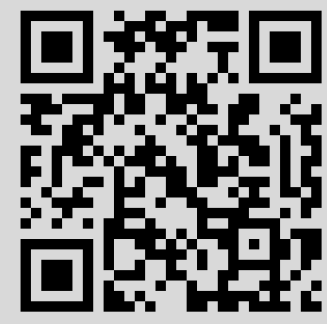




\section{УСЛОВИЯ ИНТЕГРИРУЕМОСТИ ДЛЯ АНАЛОГОВ РЕЛЯТИВИСТСКОЙ ЦЕПОЧКИ ТОДЫ}

Рассматривается класс дискретно-дифференциальных уравнений, содержащий релятивистскую цепочку Тоды и характеризующийся одной произвольной функцией шести переменных. Получены три условия, позволяющие проверить на интегрируемость любое заданное уравнение из этого класса. При выводе этих условий используется наличие у уравнения высших симметрий, которые отличают уравнения, интегрируемые методом обратной задачи рассеяния.

Ключевые слова: релятивистская цепочка Тоды, высшие симметрии, условия интегрируемости.

\section{1. ВВЕДЕНИЕ}

Будем рассматривать дискретно-дифференциальные уравнения вида

$$
\ddot{u}_{n}=f\left(u_{n+1}, u_{n}, u_{n-1}, \dot{u}_{n+1}, \dot{u}_{n}, \dot{u}_{n-1}\right) \equiv f_{n},
$$

где $u_{n}=u_{n}(t), n \in \mathbb{Z}, \dot{u}_{n}=d u_{n} / d t, \ddot{u}_{n}=d^{2} u_{n} / d t^{2}$. Кроме того, предположим, что $\partial f_{n} / \partial \dot{u}_{n+1} \neq 0$ или $\partial f_{n} / \partial \dot{u}_{n-1} \neq 0$. Известным представителем этого класса уравнений является релятивистская цепочка Тоды [1]

$$
\ddot{u}_{n}=\frac{\dot{u}_{n+1} \dot{u}_{n}}{1+e^{u_{n}-u_{n+1}}}-\frac{\dot{u}_{n} \dot{u}_{n-1}}{1+e^{u_{n-1}-u_{n}}} .
$$

Другие примеры интегрируемых уравнений вида (1) получены в работах [2], [3], причем в последней из них при помощи симметрийного метода получен целый список таких уравнений. Симметрийный метод использует такой отличительный признак уравнений, интегрируемых методом обратной задачи рассеяния, как наличие высших симметрий и законов сохранения, и позволяет находить все интегрируемые уравнения определенного вида или проверять на интегрируемость заданное уравнение. При помощи симметрийного метода получены условия интегрируемости и полные списки интегрируемых уравнений для классов, которые включают, например, уравнение Кортевега-де Фриза, нелинейное уравнение Шредингера и цепочки

* Институт математики Уфимского центра РАН, Уфа, Россия. E-mail: RvlYamilov@matem.anrb.ru 
Тоды и Вольтерра. Перечисленные результаты вместе с описанием метода содержатся, например, в обзорах [4]-[6].

Напомним вкратце, как был получен список уравнений в работе [3]. Рассматривались уравнения вида

$$
\ddot{u}_{n}=f\left(u_{n+1}, u_{n}, \dot{u}_{n+1}, \dot{u}_{n}\right)-g\left(u_{n}, u_{n-1}, \dot{u}_{n}, \dot{u}_{n-1}\right) \equiv h_{n}
$$

и предполагалось, что они имеют симметрию

$$
u_{n, \tau}=f\left(u_{n+1}, u_{n}, \dot{u}_{n+1}, \dot{u}_{n}\right)+g\left(u_{n}, u_{n-1}, \dot{u}_{n}, \dot{u}_{n-1}\right) \equiv s_{n}
$$

(индексом $\tau$ обозначена производная по $\tau$ ), т.е. что уравнения (3) и $(4)$ совместны. Совместность означает, что эти уравнения имеют общие решения $u_{n}(t, \tau)$. Для таких решений справедливо соотношение $\partial^{3} u_{n} / \partial t^{2} \partial \tau=\partial^{3} u_{n} / \partial \tau \partial t^{2}$, которое записывается следующим образом:

$$
\begin{gathered}
D_{t}^{2} s_{n}=D_{\tau} h_{n}, \\
D_{t}=\sum_{i} \dot{u}_{n+i} \frac{\partial}{\partial u_{n+i}}+\sum_{i} h_{n+i} \frac{\partial}{\partial \dot{u}_{n+i}}, \quad D_{\tau}=\sum_{i} s_{n+i} \frac{\partial}{\partial u_{n+i}}+\sum_{i} D_{t}\left(s_{n+i}\right) \frac{\partial}{\partial \dot{u}_{n+i}} .
\end{gathered}
$$

Условие совместности уравнений (3) и (4) приводит, таким образом, к уравнению (5) для функций $f$ и $g$, которое и использовалось в работе [3] для поиска интегрируемых уравнений вида (3).

Такой подход позволяет находить новые интегрируемые примеры, но не дает естественных условий для проверки заданного уравнения на интегрируемость. Причина в том, что здесь жестко фиксируется вид симметрии. Однако интегрируемые уравнения имеют бесконечно много высших симметрий, и выбор другой формы симметрии может привести, вообще говоря, к другим результатам.

Мы будем следовать в этой работе более общей схеме симметрийного метода, которая применялась в работах [7] для иследования класса уравнений вида

$$
\dot{u}_{n}=a\left(u_{n+1}, u_{n}, u_{n-1}\right) \equiv a_{n},
$$

содержащего уравнение Вольтерра, и класса цепочки Тоды:

$$
\ddot{u}_{n}=b\left(u_{n+1}, u_{n}, u_{n-1}, \dot{u}_{n}\right) \equiv b_{n} .
$$

Согласно этой схеме используется предположение о наличии у уравнения одной или двух высших симметрий (или законов сохранения) достаточно большого порядка. Форма и порядок симметрии не фиксируются. При таком предположении выводятся несколько условий для правой части уравнения, т.е. для функций $a_{n}, b_{n}$. Такие условия не зависят от формы и порядка симметрии и легко проверяются для любого заданного уравнения.

Приведем пример простейшего условия интегрируемости и соответствующего утверждения в случае уравнений вида (6). Если уравнение (6) такое, что 
$\partial a_{n} / \partial u_{n+1} \neq 0$, имеет высшую симметрию порядка $m \geqslant 2$, то существует функция $q_{n}=q\left(u_{n+1}, u_{n}, u_{n-1}, u_{n-2}\right)$, удовлетворяющая соотношению

$$
D_{t} \ln \frac{\partial a_{n}}{\partial u_{n+1}}=q_{n+1}-q_{n}
$$

где $D_{t}=\sum_{i} a_{n+i} \partial / \partial u_{n+i}$. Что такое высшая симметрия и ее порядок и как проверить условие (8) для любой заданной функции $a_{n}$, будет обсуждаться ниже на примере уравнений вида (1).

Цель настоящей работы - вывести для цепочек вида (1) три условия интегрируемости, аналогичных соотношению (8). Мы будем следовать схеме симметрийного метода, которая разработана для классов уравнений (6), (7) в работах [7] и подробно изложена в статьях [8], [9]. При выводе этих условий возникает существенно новое препятствие теоретического характера, которое преодолевается при помощи сформулированной в разделе 3 леммы.

\section{2. НЕОБХОДИМЫЕ СВЕДЕНИЯ}

Сначала мы, следуя статьям [8] и обзору [9], приведем некоторые сведения из общей теории симметрийного метода, необходимые для изложения результатов этой работы.

Вводя функцию $v_{n}=\dot{u}_{n}$, удобно перейти от уравнения (1) к эквивалентной ему системе уравнений

$$
\dot{u}_{n}=v_{n}, \quad \dot{v}_{n}=f_{n}=f\left(u_{n+1}, u_{n}, u_{n-1}, v_{n+1}, v_{n}, v_{n-1}\right) .
$$

Векторная форма записи этой системы имеет вид

$$
\dot{U}_{n}=F_{n}=F\left(U_{n+1}, U_{n}, U_{n-1}\right), \quad U_{n}=\left(\begin{array}{c}
u_{n} \\
v_{n}
\end{array}\right), \quad F_{n}=\left(\begin{array}{c}
v_{n} \\
f_{n}
\end{array}\right) .
$$

Высшие симметрии системы (9) в векторной форме записываются следующим образом:

$$
U_{n, \tau}=G_{n}=G\left(U_{n+m}, U_{n+m-1}, \ldots, U_{n+m^{\prime}}\right), \quad G_{n}=\left(\begin{array}{c}
\varphi_{n} \\
\psi_{n}
\end{array}\right),
$$

где $m>m^{\prime}$.

Симметрия (11) является совместным с (10) уравнением, т.е. эти уравнения имеют общие решения $U_{n}(t, \tau)$. Равенство $\partial^{2} U_{n} / \partial t \partial \tau=\partial^{2} U_{n} / \partial \tau \partial t$ записывается на таких решениях в виде $D_{t} G_{n}=D_{\tau} F_{n}$, где $D_{t}$ и $D_{\tau}$ - операторы дифференцирования в силу (10) и (11). Полученное соотношение можно представить следующим образом:

$$
\sum_{i=m^{\prime}}^{m} \frac{\partial G_{n}}{\partial U_{n+i}} F_{n+i}=\sum_{i=-1}^{1} \frac{\partial F_{n}}{\partial U_{n+i}} G_{n+i}
$$

где $\partial G_{n} / \partial U_{n+i}-$ матрицы вида

$$
\frac{\partial G_{n}}{\partial U_{n+i}}=\left(\begin{array}{ll}
\partial \varphi_{n} / \partial u_{n+i} & \partial \varphi_{n} / \partial v_{n+i} \\
\partial \psi_{n} / \partial u_{n+i} & \partial \psi_{n} / \partial v_{n+i}
\end{array}\right)
$$


матрицы $\partial F_{n} / \partial U_{n+i}$ определяются аналогично. Функции $u_{n+i}, v_{n+i}$ рассматриваются в (12) как независимые переменные, поэтому соотношение (12), которое является условием совместности уравнений (10) и (11), налагает сильные ограничения на вектор-функции $F_{n}$ и $G_{n}$.

Высшей симметрией уравнения (10) (или системы (9)) мы будем называть далее уравнение вида (11) с $m>m^{\prime}$, правая часть $G_{n}$ которого удовлетворяет условию совместности (12). Число $m$ называется порядком симметрии, которая является невырожденной, если $\operatorname{det}\left(\partial G_{n} / \partial U_{n+m}\right) \neq 0$. Используя высшие симметрии, можно строить приближенные решения $L_{n}$ уравнения

$$
\dot{L}_{n}=\left[F_{n}^{*}, L_{n}\right]
$$

играющего важную роль в дальнейшем. Поэтому мы переходим к подробному обсуждению этого уравнения.

Определим производные Фреше от вектор-функций $F_{n}, G_{n}$ :

$$
F_{n}^{*}=\sum_{i=-1}^{1} \frac{\partial F_{n}}{\partial U_{n+i}} T^{i}, \quad G_{n}^{*}=\sum_{i=m^{\prime}}^{m} \frac{\partial G_{n}}{\partial U_{n+i}} T^{i} .
$$

Здесь $T^{i}$ - степени оператора сдвига $T: n \rightarrow n+1$, например, $T^{i} F_{n}=F_{n+i}=$ $F\left(U_{n+i+1}, U_{n+i}, U_{n+i-1}\right)$. Вводя обозначения

$$
f_{n}^{(i)}=\frac{\partial f_{n}}{\partial u_{n+i}}, \quad g_{n}^{(i)}=\frac{\partial f_{n}}{\partial v_{n+i}}
$$

для производных функции $f_{n}$ из системы (9), получаем более подробную формулу:

$$
F_{n}^{*}=\left(\begin{array}{cc}
0 & 0 \\
f_{n}^{(1)} & g_{n}^{(1)}
\end{array}\right) T+\left(\begin{array}{cc}
0 & 1 \\
f_{n}^{(0)} & g_{n}^{(0)}
\end{array}\right)+\left(\begin{array}{cc}
0 & 0 \\
f_{n}^{(-1)} & g_{n}^{(-1)}
\end{array}\right) T^{-1}
$$

Решения уравнения (13) - формальные ряды вида

$$
L_{n}=\sum_{i=-\infty}^{k} l_{n}^{(i)} T^{i}
$$

коэффициенты $l_{n}^{(i)}$ которых являются $(2 \times 2)$-матрицами. Ряды умножаются по правилу $\left(l_{n} T^{i}\right)\left(\hat{l}_{n} T^{j}\right)=l_{n} \hat{l}_{n+i} T^{i+j}$, а $\left[F_{n}^{*}, L_{n}\right]=F_{n}^{*} L_{n}-L_{n} F_{n}^{*}$ - стандартный коммутатор. Наконец, $\dot{L}_{n}=\sum_{i=-\infty}^{k} i_{n}^{(i)} T^{i}$, т.е. коэффициенты дифференцируются по времени в силу уравнения (10).

Если $\operatorname{det} l_{n}^{(k)} \neq 0$, то формальный ряд (16) можно обратить, используя стандартное определение: $L_{n}^{-1} L_{n}=L_{n} L_{n}^{-1}=E$, где $E=E T^{0}$ - оператор умножения на единичную матрицу $E$. Обратный ряд имеет вид $L_{n}^{-1}=\sum_{i=-\infty}^{-k} \hat{l}_{n}^{(i)} T^{i}$, и первые коэффициенты находятся по формулам

$$
\hat{l}_{n}^{(-k)}=\left(l_{n-k}^{(k)}\right)^{-1}, \quad \hat{l}_{n}^{(-k-1)}=-\left(l_{n-k}^{(k)}\right)^{-1} l_{n-k}^{(k-1)}\left(l_{n-k-1}^{(k)}\right)^{-1} .
$$


Оператор $G_{n}^{*}$ можно считать частным случаем ряда $(16)$ с $k=m$ и $l_{n}^{(i)}=0$ при $i<m^{\prime}$. Если симметрия (11) невырожденна, то можно найти формальный ряд $\left(G_{n}^{*}\right)^{-1}$, который используется ниже.

Мы будем иметь дело с рядом (16) порядка $k=1$ с невырожденным старшим коэффициентом:

$$
L_{n}=\sum_{i \leqslant 1} l_{n}^{(i)} T^{i}, \quad \operatorname{det} l_{n}^{(1)} \neq 0
$$

который является приближенным решением длины $m$ уравнения (13). Вообще говоря, для ряда (17) имеет место формула

$$
\dot{L}_{n}-\left[F_{n}^{*}, L_{n}\right]=\sum_{i \leqslant 2} \theta_{n}^{(i)} T^{i} .
$$

Формальный ряд (17) называется приближенным решением длины $m \geqslant 1$ уравнения (13), если $m$ старших коэффициентов ряда (18) равняются нулю: $\theta_{n}^{(i)}=0$ при $2 \geqslant i \geqslant 3-m$. Можно доказать следующее утверждение.

Теорема 1. Если уравнение вида (10) имеет две невырожденные высшие симметрии $U_{n, \tau}=G_{n}$ и $U_{n, \hat{\tau}}=\widehat{G}_{n}$ порядков $m \geqslant 1 u m+1$, то соответствующее ему уравнение (13) обладает приближенным решением длины $m$, которое имеет вид (17) и строится по формуле $L_{n}=\widehat{G}_{n}^{*}\left(G_{n}^{*}\right)^{-1}$.

Сделаем несколько замечаний о мотивировке предположений, использованных в этой теореме. Предположение о порядках $m \geqslant 1$ и $m+1$ высших симметрий использовалось и прежде в случае аналогов (6) уравнения Вольтерра, чтобы получить решение (17) первого порядка. Законность такого предположения обосновывалась следующим образом: мы рассматриваем цепочки, подобные уравнению Вольтерра, имеющему симметрию любого порядка $m \geqslant 1$. Понятие невырожденности симметрии в этом скалярном случае не имеет смысла. Можно проверить, что релятивистская цепочка Тоды (2) имеет для любого порядка $m \geqslant 1$ как вырожденную, так и невырожденную высшую симметрию (см., например, работу [9]). Поэтому, рассматривая уравнения такого типа, мы можем использовать дополнительное предположение о невырожденности симметрий, чтобы обратить оператор $G_{n}^{*}$. В случае классической цепочки Тоды и уравнений (7) ситуация аналогична, однако условия невырожденности удается избежать, поскольку оператор $G_{n}^{*}$ обращается для любого вида симметрий.

Далее мы используем вместо решений $G_{n}$ уравнения (12) приближенные решения (17) уравнения (13). Вычисляя коэффициенты $l_{n}^{(i)}$, мы будем получать условия интегрируемости для функции $f_{n}$, определяющей систему (9). Важно отметить, что так как в соответствии с теоремой 1 мы строим формальный ряд $L_{n}$ по правым частям уравнений (11), то элементы матриц $l_{n}^{(i)}$ будут функциями вида

$$
\phi_{n}=\phi\left(u_{n+k_{1}}, v_{n+k_{2}}, u_{n+k_{1}-1}, v_{n+k_{2}-1}, \ldots, u_{n+k_{1}^{\prime}}, v_{n+k_{2}^{\prime}}\right)
$$

с любыми целыми $k_{i}, k_{i}^{\prime}: k_{1} \geqslant k_{1}^{\prime}, k_{2} \geqslant k_{2}^{\prime}$. 
Перечислим преимущества уравнения (13) по сравнению с уравнением (12). Поскольку мы рассматриваем решения вида (17), то вычисления становятся проще, а в полученных условиях интегрируемости не будет зависимости от порядка $m$ высшей симметрии. Приближенные решения уравнения (13) не только образуют линейное пространство, но их можно перемножать. Наконец, при помощи решений этого уравнения можно строить для системы (9) локальные законы сохранения, которые имеют вид соотношений $D_{t} p_{n}=(T-1) q_{n}$, где $p_{n}$ и $q_{n}-$ функции вида $(19)\left(p_{n}\right.$ называется плотностью закона сохранения).

Действительно, для решения (17) уравнения (13) имеет место формула

$$
D_{t} \operatorname{tr} \operatorname{res} L_{n}^{j} \in \operatorname{Im}(T-1)
$$

(имеется в виду, что указанная функция принадлежит образу оператора $T-1$ ). Здесь $L_{n}^{j}=\sum_{i \leqslant j} \tilde{l}_{n}^{(i)} T^{i}$, и вычет этого формального ряда определяется как коэффициент при $T^{0}$ : res $L_{n}^{j}=\tilde{l}_{n}^{(0)}$. Формула (20) означает, что след матрицы $\tilde{l}_{n}^{(0)}$ является плотностью локального закона сохранения системы (9) (функцию $q_{n}$ легко построить по известной плотности). Если (17) - приближенное решение длины $m \geqslant 3$, то формулу (20) можно использовать при степенях $1 \leqslant j \leqslant m-2$.

\section{3. ОСНОВНАЯ ТРУДНОСТЬ}

Здесь мы выведем из (13), (17) два условия интегрируемости для систем вида (9) и увидим, в чем заключается основная проблема, с которой мы затем справимся при помощи леммы. Будет учитываться только одно ограничение:

$$
g_{n}^{(1)}=\frac{\partial f_{n}}{\partial v_{n+1}} \neq 0
$$

(см. (14)). Симметричный случай $g_{n}^{(-1)} \neq 0$ сводится к неравенству (21), так как замена переменных $\tilde{u}_{n}=u_{-n}, \tilde{v}_{n}=v_{-n}$ переводит симметрии системы (9) в симметрии, и интегрируемость системы таким образом не нарушается.

Введем в дополнение к формулам (15) и (17) обозначения

$$
F_{n}^{*}=F_{n}^{(1)} T+F_{n}^{(0)}+F_{n}^{(-1)} T^{-1}, \quad l_{n}^{(i)}=\left(\begin{array}{cc}
a_{n}^{(i)} & b_{n}^{(i)} \\
c_{n}^{(i)} & d_{n}^{(i)}
\end{array}\right) .
$$

Предположим, что (17) - приближенное решение уравнения (13) длины $m \geqslant 2$. Тогда можно использовать соотношения $\theta_{n}^{(2)}=0$ и $\theta_{n}^{(1)}=0$ (см. (18)), которые записываются в виде

$$
\begin{gathered}
F_{n}^{(1)} l_{n+1}^{(1)}=l_{n}^{(1)} F_{n+1}^{(1)}, \\
i_{n}^{(1)}=F_{n}^{(1)} l_{n+1}^{(0)}+F_{n}^{(0)} l_{n}^{(1)}-l_{n}^{(1)} F_{n+1}^{(0)}-l_{n}^{(0)} F_{n}^{(1)} .
\end{gathered}
$$

Соотношение (23) дает три уравнения для элементов матрицы $l_{n}^{(1)}$, одно из которых имеет вид $b_{n}^{(1)} g_{n+1}^{(1)}=0$. Из условия $(21)$ следует, что $b_{n}^{(1)}=0$, и так как $\operatorname{det} l_{n}^{(1)}=a_{n}^{(1)} d_{n}^{(1)}$, то с учетом (17) мы имеем

$$
b_{n}^{(1)}=0, \quad a_{n}^{(1)} \neq 0, \quad d_{n}^{(1)} \neq 0 .
$$


Еще одно из уравнений $(23)$ записывается как $g_{n}^{(1)} d_{n+1}^{(1)}=d_{n}^{(1)} g_{n+1}^{(1)}$. Представляя его в виде $(T-1)\left(d_{n}^{(1)} / g_{n}^{(1)}\right)=0$ и учитывая, что ядро оператора $T-1$ состоит из постоянных функций, получаем формулу $d_{n}^{(1)}=\alpha g_{n}^{(1)}$ с константой $\alpha \neq 0$. Наконец, деля соотношение (18) на $\alpha$, мы показываем для уравнения (13) существование приближенного решения вида (17) длины $m \geqslant 2$ :

$$
d_{n}^{(1)}=g_{n}^{(1)} .
$$

Это решение и используется далее.

Левые нижние элементы в соотношении (23) и правые верхние в (24) дают формулы для функций $c_{n}^{(1)}$ и $b_{n}^{(0)}$ :

$$
b_{n}^{(0)}=1-\frac{a_{n}^{(1)}}{g_{n}^{(1)}}, \quad c_{n}^{(1)}=f_{n}^{(1)}-a_{n}^{(1)} \varrho_{n}, \quad \varrho_{n}=\frac{f_{n-1}^{(1)}}{g_{n-1}^{(1)}} .
$$

Наконец, из диагональной части (24) получаем уравнения для $a_{n}^{(1)}$ и $d_{n}^{(0)}$ :

$$
\begin{gathered}
D_{t} \ln a_{n}^{(1)}=(T-1) \varrho_{n}, \\
D_{t} \ln g_{n}^{(1)}=(T-1)\left(d_{n}^{(0)}-g_{n}^{(0)}-\frac{a_{n}^{(1)} \varrho_{n}}{g_{n}^{(1)}}\right),
\end{gathered}
$$

причем действие оператора $D_{t}$ на функциях (19) задается как

$$
D_{t}=\sum_{i} v_{n+i} \frac{\partial}{\partial u_{n+i}}+\sum_{i} f_{n+i} \frac{\partial}{\partial v_{n+i}} .
$$

Итак, мы получили два условия для функции $f_{n}$ из (9), которые формулируются следующим образом: существуют функции $a_{n}^{(1)}$ и $d_{n}^{(0)}$ вида (19), удовлетворяющие соотношениям (28) и (29). Эти условия имеют смысл, если помнить о том, что функции $u_{n+i}, v_{n+i}$ рассматриваются здесь как независимые переменные. Условия интегрируемости имеют вид локальных законов сохранения, причем (29) аналогично условию (8). При заданной системе (9) мы знаем левую часть (29) и можем проверить, принадлежит ли она $\operatorname{Im}(T-1)$ (в следующем разделе объясняется, как это сделать). Если принадлежит, то мы можем найти линейную комбинацию $d_{n}^{(0)}-a_{n}^{(1)} \varrho_{n} / g_{n}^{(1)}$ неизвестных функций $d_{n}^{(0)}, a_{n}^{(1)}$. В случае уравнений вида (6) и $(7)$ все условия интегрируемости аналогичны (29) и, если уравнение интегрируемо, позволяют найти все коэффициенты формального ряда (17).

В уравнении (28), напротив, правая часть известна, а плотность закона сохранения $\ln a_{n}^{(1)}$ - неизвестная функция. Мы не умеем проверять такие условия интегрируемости для заданной функции $f_{n}$ и не можем найти $a_{n}^{(1)}$. Соотношения для остальных функций $a_{n}^{(i)}, d_{n}^{(i)}$ аналогичны (28), (29). Мы не можем найти функции $a_{n}^{(i)}$, а так как они входят во все соотношения, то все эти условия интегрируемости становятся нерабочими. Это и есть та трудность, которая возникает при применении к системам (9), а значит, и к уравнениям (1) стандартной схемы симметрийного метода. 
Эту трудность удается преодолеть при помощи более простого, чем (17), приближенного решения уравнения (13). При его построении используется некое точное решение $\Lambda_{n}$, которое существует для любой функциии $f_{n}$ и является в этом смысле тривиальным. Оператор умножения на единичную матрицу $E$ - очевидное тривиальное решение уравнения (13). Формальный ряд $\Lambda_{n}$ оказывается квадратным корнем из этого оператора.

Лемма. Существует единственное решение $\Lambda_{n}$ уравнения (13) такое, что

$$
\Lambda_{n}=\sum_{i \leqslant 0} \lambda_{n}^{(i)} T^{i}, \quad \lambda_{n}^{(i)}=\left(\begin{array}{cc}
\alpha_{n}^{(i)} & \beta_{n}^{(i)} \\
\gamma_{n}^{(i)} & \delta_{n}^{(i)}
\end{array}\right), \quad \lambda_{n}^{(0)}=\left(\begin{array}{cc}
1 & 0 \\
\gamma_{n}^{(0)} & -1
\end{array}\right), \quad \Lambda_{n}^{2}=E .
$$

ДокАзАтельство (сокращенное). Разложим $\Lambda_{n}$ на диагональную и антидиагональную части:

$$
\begin{gathered}
\Lambda_{n}=\sigma+R_{n}+S_{n}, \quad \sigma=\left(\begin{array}{cc}
1 & 0 \\
0 & -1
\end{array}\right) \\
R_{n}=\sum_{i \leqslant-1} r_{n}^{(i)} T^{i}=\sum_{i \leqslant-1}\left(\begin{array}{cc}
\alpha_{n}^{(i)} & 0 \\
0 & \delta_{n}^{(i)}
\end{array}\right) T^{i}, \quad S_{n}=\sum_{i \leqslant 0} s_{n}^{(i)} T^{i}=\sum_{i \leqslant 0}\left(\begin{array}{cc}
0 & \beta_{n}^{(i)} \\
\gamma_{n}^{(i)} & 0
\end{array}\right) T^{i},
\end{gathered}
$$

где $\beta_{n}^{(0)}=0$, и рассмотрим сначала условие $\Lambda_{n}^{2}=E$. Оно эквивалентно двум соотношениям:

$$
2 \sigma R_{n}+R_{n}^{2}+S_{n}^{2}=0, \quad R_{n} S_{n}+S_{n} R_{n}=0
$$

Рассмотрим первое из них как уравнение для формального ряда $R_{n}$. Вводя обозначения

$$
\chi_{n}^{(-1)}=0, \quad \chi_{n}^{(k)}=\sum_{k+1 \leqslant i \leqslant-1}\left(r_{n}^{(i)} r_{n+i}^{(k-i)}+s_{n}^{(i)} s_{n+i}^{(k-i)}\right), \quad k \leqslant-2,
$$

и собирая в этом уравнении коэффициенты при одинаковых степенях $T$, получаем

$$
2 \sigma r_{n}^{(k)}+\chi_{n}^{(k)}+s_{n}^{(0)} s_{n}^{(k)}+s_{n}^{(k)} s_{n+k}^{(0)}=0, \quad k \leqslant-1 .
$$

Отсюда для любого $k \leqslant-1$ функции $\alpha_{n}^{(k)}, \delta_{n}^{(k)}$ явно выражаются через $\beta_{n}^{(-1)}, \beta_{n}^{(-2)}, \ldots, \beta_{n}^{(k)}$ и $\gamma_{n}^{(0)}, \gamma_{n}^{(-1)}, \ldots, \gamma_{n}^{(k+1)}$, и $R_{n}$ однозначно определяется.

То же самое уравнение позволяет получить представление вида $R_{n}=\sigma \sum_{i \geqslant 1} c_{i} S_{n}^{2 i}$ с постоянными коэффициентами $c_{i}$. Так как $\left(s_{n}^{(0)}\right)^{2}=0$, то $S_{n}^{2 i}=\sum_{j \leqslant-i} \tilde{s}_{n}^{(j)} T^{j}$, поэтому такое представление определено корректно. Собирая в первом из уравнений (33) коэффициенты при одинаковых степенях $S_{n}$, получаем формулы для постоянных $c_{i}$ :

$$
2 c_{1}+1=0, \quad 2 c_{k}+\sum_{1 \leqslant i \leqslant k-1} c_{i} c_{k-i}=0, \quad k \geqslant 2 .
$$


Теперь мы имеем равенство

$$
R_{n} S_{n}+S_{n} R_{n}=\sigma\left[\sum_{i \geqslant 1} c_{i} S_{n}^{2 i}, S_{n}\right]
$$

которое показывает, что $R_{n}$ удовлетворяет и второму из уравнений (33).

Нам осталось использовать уравнение (13), т.е. $\Omega_{n}=\dot{\Lambda}_{n}-\left[F_{n}^{*}, \Lambda_{n}\right]=0$. Введем обозначения для диагональной $\Omega_{n}^{\|}=0$ и антидиагональной $\Omega_{n}^{\perp}=0$ частей этого уравнения, а также для следующих операторов и формальных рядов:

$$
\begin{aligned}
f_{n}^{*, u}=f_{n}^{(1)} T+f_{n}^{(0)}+f_{n}^{(-1)} T^{-1}, & f_{n}^{*, v}=g_{n}^{(1)} T+g_{n}^{(0)}+g_{n}^{(-1)} T^{-1}, \\
A_{n}=\sum_{i \leqslant-1} \alpha_{n}^{(i)} T^{i}, \quad B_{n}=\sum_{i \leqslant-1} \beta_{n}^{(i)} T^{i}, & C_{n}=\sum_{i \leqslant 0} \gamma_{n}^{(i)} T^{i}, \quad D_{n}=\sum_{i \leqslant-1} \delta_{n}^{(i)} T^{i} .
\end{aligned}
$$

Тогда уравнение $\Omega_{n}^{\perp}=0$ запишется в виде системы

$$
\begin{gathered}
B_{n} f_{n}^{*, v}+\dot{B}_{n}+A_{n}-D_{n}+2=0 \\
f_{n}^{*, v} C_{n}-\dot{C}_{n}+f_{n}^{*, u} A_{n}-D_{n} f_{n}^{*, u}+2 f_{n}^{*, u}=0 .
\end{gathered}
$$

Собирая коэффициенты при одинаковых степенях $T$ и используя неравенство (21), нетрудно получить отсюда явные рекуррентные формулы для коэффициентов $B_{n}$ и $C_{n}$, которые однозначно определяют формальный ряд $S_{n}$.

Осталось показать, что при определенных таким образом формальных рядах $R_{n}$ и $S_{n}$ последнее условие $\Omega_{n}^{\|}=0$ удовлетворяется автоматически. Так как $E-$ решение уравнения (13), $\Lambda_{n}^{2}=E$ и $\Omega_{n}^{\perp}=0$, то

$$
\dot{E}-\left[F_{n}^{*}, E\right]=D_{t}\left(\Lambda_{n}^{2}\right)-\left[F_{n}^{*}, \Lambda_{n}^{2}\right]=\Lambda_{n} \Omega_{n}+\Omega_{n} \Lambda_{n}=\Lambda_{n} \Omega_{n}^{\|}+\Omega_{n}^{\|} \Lambda_{n}=0 .
$$

Если $\Omega_{n}^{\|} \neq 0$, то имеется представление $\Omega_{n}^{\|}=\sum_{i \leqslant l} \omega_{n}^{(i)} T^{i}$, где $\omega_{n}^{(i)}-$ диагональные матрицы и $\omega_{n}^{(l)} \neq 0$. Однако, собирая коэффициенты при $T^{l}$ в последнем из равенств (36) и рассматривая диагональную часть полученного соотношения, приходим к противоречию: $\sigma \omega_{n}^{(l)}+\omega_{n}^{(l)} \sigma=2 \sigma \omega_{n}^{(l)}=0$. Лемма доказана.

Итак, коэффициенты решения $\Lambda_{n}$ находятся следующим образом. Соотношения (34) позволяют выразить функции $\alpha_{n}^{(i)}, \delta_{n}^{(i)}$ через $\beta_{n}^{(i)}, \gamma_{n}^{(i)}$, например,

$$
\begin{aligned}
& \alpha_{n}^{(-1)}=-\frac{1}{2} \beta_{n}^{(-1)} \gamma_{n-1}^{(0)}, \quad \delta_{n}^{(-1)}=\frac{1}{2} \gamma_{n}^{(0)} \beta_{n}^{(-1)}, \\
& \alpha_{n}^{(-2)}=-\frac{1}{2}\left(\alpha_{n}^{(-1)} \alpha_{n-1}^{(-1)}+\beta_{n}^{(-1)} \gamma_{n-1}^{(-1)}+\beta_{n}^{(-2)} \gamma_{n-2}^{(0)}\right), \\
& \delta_{n}^{(-2)}=\frac{1}{2}\left(\delta_{n}^{(-1)} \delta_{n-1}^{(-1)}+\gamma_{n}^{(-1)} \beta_{n-1}^{(-1)}+\gamma_{n}^{(0)} \beta_{n}^{(-2)}\right) .
\end{aligned}
$$


Затем из системы (35) получаем рекуррентные формулы для $\beta_{n}^{(i)}, \gamma_{n}^{(i)}$, например,

$$
\begin{aligned}
& \beta_{n}^{(-1)}=-\frac{2}{g_{n-1}^{(1)}}, \quad \gamma_{n}^{(0)}=-\frac{2 f_{n-1}^{(1)}}{g_{n-1}^{(1)}}, \\
& \beta_{n}^{(-2)}=-\frac{\beta_{n}^{(-1)} g_{n-1}^{(0)}+\dot{\beta}_{n}^{(-1)}+\alpha_{n}^{(-1)}-\delta_{n}^{(-1)}}{g_{n-2}^{(1)}}, \\
& \gamma_{n}^{(-1)}=-\frac{g_{n-1}^{(0)} \gamma_{n-1}^{(0)}-\dot{\gamma}_{n-1}^{(0)}+f_{n-1}^{(1)} \alpha_{n}^{(-1)}-\delta_{n-1}^{(-1)} f_{n-2}^{(1)}+2 f_{n-1}^{(0)}}{g_{n-1}^{(1)}} .
\end{aligned}
$$

Отсюда функции $\beta_{n}^{(i)}, \gamma_{n}^{(i)}$ выражаем через $f_{n}^{(i)}, g_{n}^{(i)}$, заданные формулами (14), т.е. через функцию $f_{n}$, которая определяет систему (9).

Теперь, используя формальный ряд $\Lambda_{n}$ из леммы, можно построить следующие точные решения уравнения (13):

$$
\Lambda_{n}^{+}=\frac{1}{2}\left(E+\Lambda_{n}\right), \quad \Lambda_{n}^{-}=\frac{1}{2}\left(E-\Lambda_{n}\right) .
$$

Затем при помощи формального ряда $L_{n}$ из теоремы 1 можно ввести два приближенных решения уравнения (13)

$$
L_{n}^{+}=\Lambda_{n}^{+} L_{n} \Lambda_{n}^{+}, \quad L_{n}^{-}=\Lambda_{n}^{-} L_{n} \Lambda_{n}^{-},
$$

которые имеют те же порядок 1 и длину $m$. Решения (40) позволяют преодолеть указанные выше трудности и отделить удобные рабочие условия интегрируемости от нерабочих.

\section{4. УСЛОВИЯ ИНТЕГРИРУЕМОСТИ}

Рассмотрим сначала более важный случай приближенного решения $L_{n}^{-}$уравнения (13) с длиной $m \geqslant 4$. Оказывается, что теперь в отличие от решения (17) мы будем получать только условия интегрируемости, аналогичные (8), которые являются рабочими.

Из формул (30) и (39) имеем

$$
\Lambda_{n}^{-}=\left(\begin{array}{cc}
0 & 0 \\
-\gamma_{n}^{(0)} / 2 & 1
\end{array}\right)-\frac{1}{2} \lambda_{n}^{(-1)} T^{-1}-\frac{1}{2} \lambda_{n}^{(-2)} T^{-2}-\ldots
$$

Используем те же обозначения $L_{n}^{-}=\sum_{i \leqslant 1} l_{n}^{(i)} T^{i}$ и $(22)$, что для формального ряда (17). Из выражений (40) видно, что функции $b_{n}^{(1)}, d_{n}^{(1)}$ для $L_{n}, L_{n}^{-}$совпадают, т.е. $b_{n}^{(1)}=0, d_{n}^{(1)}=g_{n}^{(1)} \neq 0$ (см. (21), (25), (26)). Другие коэффициенты формального ряда $L_{n}^{-}$удобно определять при помощи уравнения $\Lambda_{n}^{-} L_{n}^{-} \Lambda_{n}^{-}=L_{n}^{-}$, которое следует из свойства $\left(\Lambda_{n}^{-}\right)^{2}=\Lambda_{n}^{-}$. Из него находим, например,

$$
a_{n}^{(1)}=0, \quad c_{n}^{(1)}=f_{n}^{(1)}, \quad b_{n}^{(0)}=1, \quad a_{n}^{(0)}=\varrho_{n}
$$


с $\varrho_{n}$, заданным в $(27)$. Аналогично можно выразить любую из функций $a_{n}^{(i)}, b_{n}^{(i)}$, $c_{n}^{(i)}$ через $f_{n}$ и $d_{n}^{(i)}$ с $i \leqslant 0$.

Уравнение (13) используется только для определения функций $d_{n}^{(i)}$. Так как $L_{n}^{-}$ является решением длины $m \geqslant 4$, то мы получаем соотношения для $d_{n}^{(0)}, d_{n}^{(-1)}, d_{n}^{(-2)}$. Все они записываются в виде локальных законов сохранения, причем в случае $d_{n}^{(-1)}$, $d_{n}^{(-2)}$ - при помощи формулы $(20)$ с $j=1,2$. В результате мы получаем уравнения вида

$$
D_{t} p_{n}^{(i)}=(T-1) q_{n}^{(i)}, \quad i=1,2,3 .
$$

Здесь $p_{n}^{(i)}, q_{n}^{(i)}$ - функции вида (19), которые выражаются через $f_{n}$; кроме того, $q_{n}^{(1)}$, $p_{n}^{(2)}$ выражаются через $d_{n}^{(0)}$, функции $q_{n}^{(2)}, p_{n}^{(3)}$ - через $d_{n}^{(0)}, d_{n}^{(-1)}$, а $q_{n}^{(3)}$ - через $d_{n}^{(0)}$, $d_{n}^{(-1)}, d_{n}^{(-2)}$. Простейшие из таких формул имеют вид

$$
p_{n}^{(1)}=\ln g_{n}^{(1)}, \quad q_{n}^{(1)}=d_{n}^{(0)}-g_{n}^{(0)}, \quad p_{n}^{(2)}=\operatorname{tr} \operatorname{res} L_{n}^{-}=d_{n}^{(0)}+\varrho_{n} .
$$

В окончательных формулах удобно перейти от функций $d_{n}^{(i)}$ к $q_{n}^{(i)}$. Кроме того, нам нужны условия интегрируемости для уравнений (1), поэтому мы заменим $v_{n}$ на $\dot{u}_{n}$. Теперь $f_{n}-$ функция из $(1), D_{t}=\sum_{i} \dot{u}_{n+i} \partial / \partial u_{n+i}+\sum_{i} f_{n+i} \partial / \partial \dot{u}_{n+i}$ и мы используем обозначения

$$
\varrho_{n}=\frac{\partial f_{n-1}}{\partial u_{n}}\left(\frac{\partial f_{n-1}}{\partial \dot{u}_{n}}\right)^{-1}, \quad \omega_{n}=\frac{\partial f_{n}}{\partial u_{n}}-\frac{\partial f_{n}}{\partial \dot{u}_{n}} \varrho_{n}-\varrho_{n}^{2}+D_{t} \varrho_{n} .
$$

Формулы для плотностей законов сохранения принимают вид

$p_{n}^{(1)}=\ln \frac{\partial f_{n}}{\partial \dot{u}_{n+1}}, \quad p_{n}^{(2)}=q_{n}^{(1)}+\frac{\partial f_{n}}{\partial \dot{u}_{n}}+\varrho_{n}, \quad p_{n}^{(3)}=q_{n}^{(2)}+\frac{1}{2}\left(p_{n}^{(2)}\right)^{2}+\frac{\partial f_{n}}{\partial \dot{u}_{n-1}} \frac{\partial f_{n-1}}{\partial \dot{u}_{n}}+\omega_{n}$.

Соотношения (41), (43) являются следствием интегрируемости уравнения вида (1). Поэтому необходимые условия интегрируемости уравнения (1) формулируются так: должны существовать функции $q_{n}^{(1)}, q_{n}^{(2)}$ и $q_{n}^{(3)}$, зависящие от конечного числа переменных

$$
u_{n}, \dot{u}_{n}, u_{n+1}, \dot{u}_{n+1}, u_{n-1}, \dot{u}_{n-1}, u_{n+2}, \dot{u}_{n+2}, u_{n-2}, \dot{u}_{n-2}, \ldots,
$$

которые удовлетворяют соотношениям (41), (43). Иными словами, функции $q_{n}^{(i)}$ имеют вид (19) с $v_{n}=\dot{u}_{n}$. Функции (44) рассматриваются в соотношениях (41), (43) как независимые переменные, поэтому эти условия интегрируемости налагают сильные ограничения на функцию $f_{n}$.

В случае решения $L_{n}^{+}$, заданного формулами (39) и (40), аналогичные соображения дают $b_{n}^{(1)}=0, a_{n}^{(1)} \neq 0$. Другие коэффициенты $L_{n}^{+}$удобно находить при помощи уравнения $\Lambda_{n}^{+} L_{n}^{+} \Lambda_{n}^{+}=L_{n}^{+}$, например,

$$
d_{n}^{(1)}=0, \quad c_{n}^{(1)}=-a_{n}^{(1)} \varrho_{n}, \quad b_{n}^{(0)}=-\frac{a_{n}^{(1)}}{g_{n}^{(1)}}, \quad d_{n}^{(0)}=\frac{a_{n}^{(1)} \varrho_{n}}{g_{n}^{(1)}}
$$

с $\varrho_{n}$, определенным в (27). При помощи этого уравнения выражаем, если нужно, любую из функций $b_{n}^{(i)}, c_{n}^{(i)}, d_{n}^{(i)}$ в терминах $f_{n}, a_{n}^{(i)}$. 
Чтобы получить из (13) уравнения для функций $a_{n}^{(1)}$ и $a_{n}^{(0)}$, используем приближенное решение $L_{n}^{+}$длины $m \geqslant 3$. Как и прежде, запишем эти уравнения в виде законов сохранения

$$
D_{t} \hat{p}_{n}^{(i)}=(T-1) \hat{q}_{n}^{(i)}, \quad i=1,2,
$$

применяя в одном случае формулу (20) с $j=1$. Наконец, переходя от неизвестных функций $a_{n}^{(i)}$ к $\hat{p}_{n}^{(i)}$ и от переменной $v_{n}$ к $\dot{u}_{n}$, мы получаем уравнения (45) с

$$
\hat{q}_{n}^{(1)}=\varrho_{n}, \quad \hat{q}_{n}^{(2)}=\omega_{n}\left(\frac{\partial f_{n}}{\partial \dot{u}_{n+1}}\right)^{-1} e^{\hat{p}_{n}^{(1)}} .
$$

Здесь, как и в случае условий $(41)$, (43), функция $f_{n}$ и оператор $D_{t}$ соответствуют уравнению (1) и использованы обозначения (42). На этот раз необходимые условия интегрируемости требуют существования функций $\hat{p}_{n}^{(1)}$ и $\hat{p}_{n}^{(2)}$, которые зависят от конечного числа переменных (44) и удовлетворяют уравнениям (45), (46).

С учетом теоремы 1 главный результат работы можно сформулировать в виде следующей теоремы (напомним, что высшие симметрии определялись для уравнения (1), записанного в эквивалентной форме (9)).

Теорема 2. Если уравнение вида (1) такое, что $\partial f_{n} / \partial \dot{u}_{n+1} \neq 0$, имеет две невырожденные высшие симметрии порядков $m \geqslant 4 u m+1$, то существуют функиии $q_{n}^{(i)}$ c $i=1,2,3$ и $\hat{p}_{n}^{(i)}$ c $i=1,2$ конечного числа переменных (44), которые удовлетворяют условиям (41), (43) и (45), (46).

Как уже говорилось, мы не умеем проверять условия (45), (46). Они выписаны для полноты картины и, возможно, окажутся полезными в будущем. Для проверки интегрируемости уравнений (1) предлагается использовать три условия (41), (43).

Проверка этих условий интегрируемости для заданного уравнения (1) проводится согласно общей схеме, изложенной в работах [8], [9]. Мы сформулируем только основное утверждение этой схемы для функции самого общего вида

$$
\varphi_{n}=\varphi\left(u_{n+k_{1}}, \dot{u}_{n+k_{2}}, u_{n+k_{1}-1}, \dot{u}_{n+k_{2}-1}, \ldots, u_{n+k_{1}^{\prime}}, \dot{u}_{n+k_{2}^{\prime}}\right),
$$

где $k_{1} \geqslant k_{1}^{\prime}$ и $k_{2} \geqslant k_{2}^{\prime}$, используя формальные вариационные производные

$$
\frac{\delta \varphi_{n}}{\delta u_{n}}=\sum_{i=-k_{1}}^{-k_{1}^{\prime}} \frac{\partial \varphi_{n+i}}{\partial u_{n}}, \quad \frac{\delta \varphi_{n}}{\delta \dot{u}_{n}}=\sum_{i=-k_{2}}^{-k_{2}^{\prime}} \frac{\partial \varphi_{n+i}}{\partial \dot{u}_{n}} .
$$

Функция $\varphi_{n}$ удовлетворяет условию

$$
\frac{\delta \varphi_{n}}{\delta u_{n}}=\frac{\delta \varphi_{n}}{\delta \dot{u}_{n}}=0
$$

тогда и только тогда, когда представляется в виде

$$
\varphi_{n}=c+(T-1) \psi_{n}
$$

где $c$ - константа, а $\psi_{n}$ - функция вида (47) с (возможно) другими $k_{i}, k_{i}^{\prime}$. 
В случае, например, уравнения (41) с $i=1$ проверяем сначала условие (48) с $\varphi_{n}=D_{t} p_{n}^{(1)}$. При положительном результате нужно (и это нетрудно сделать) представить $\varphi_{n}$ в виде (49). Если $c=0$, то первое из условий интегрируемости (41) выполнено, и мы находим $q_{n}^{(1)}=\psi_{n}$. Теперь мы знаем $p_{n}^{(2)}$ и можем перейти к следующему условию интегрируемости. Понятно, что эти условия проверяются последовательно по мере нахождения функций $q_{n}^{(i)}$.

Отметим, что ядро оператора $T-1$ состоит из постоянных функций, поэтому функции $q_{n}^{(i)}$ определяются с точностью до произвольных констант $c_{i}$. Перейдем от некоторого частного решения $\left\{q_{n}^{(i)}: i=1,2,3\right\}$ системы (41), (43) к общему: $\left\{q_{n}^{(i)}+c_{i}\right\}$. При этом соответствующие плотности законов сохранения $p_{n}^{(i)}$ преобразуются следующим образом:

$$
p_{n}^{(1)} \rightarrow p_{n}^{(1)}, \quad p_{n}^{(2)} \rightarrow p_{n}^{(2)}+c_{1}, \quad p_{n}^{(3)} \rightarrow p_{n}^{(3)}+c_{1} p_{n}^{(2)}+\frac{1}{2} c_{1}^{2}+c_{2} .
$$

Отсюда с учетом линейности операторов $D_{t}$ и $T-1$ видно, что ответ на вопрос, имеет ли система (41), (43) решения, не зависит от выбора постоянных $c_{i}$. Иными словами, проверяя условия интегрируемости, мы можем выбирать функции $q_{n}^{(i)}$ как угодно.

В случае, если уравнение вида (1) удовлетворяет всем трем условиям (41), (43), мы получаем для него три локальных закона сохранения. Изложенный выше метод позволяет, если необходимо, провести дополнительную проверку уравнения на интегрируемость. Для этого мы вычисляем аналогичным образом следующие несколько коэффициентов формального ряда $L_{n}^{-}$, заданного при помощи соотношений (40). В случае положительного результата такой проверки мы также строим дополнительные законы сохранения, используя формулу (20). Следует заметить, что некоторые из полученных таким способом законов сохранения (или даже все) могут оказаться тривиальными (тривиальным называется закон сохранения, плотность которого является функцией вида (49)).

Рассмотрим в заключение пример релятивистской цепочки Тоды (2). Нетрудно проверить, что все три условия $(41),(43)$ выполнены. Так, вводя обозначения $\phi(z)=$ $1 /\left(1+e^{-z}\right)$ и $w_{n}=u_{n+1}-u_{n}$, мы имеем

$$
p_{n}^{(1)}=\ln \dot{u}_{n}+\ln \phi\left(w_{n}\right), \quad q_{n}^{(1)}=\dot{u}_{n}+\dot{u}_{n-1} \phi\left(w_{n-1}\right) .
$$

На втором этапе находим

$$
p_{n}^{(2)}=2 \dot{u}_{n}+(T-1) r_{n}, \quad q_{n}^{(2)}=2 \dot{u}_{n-1} r_{n}+D_{t} r_{n},
$$

где $r_{n}=\dot{u}_{n} \phi\left(w_{n-1}\right)$. Оказывается, можно также подобрать функции $\hat{p}_{n}^{(i)}$, удовлетворяющие соотношениям (45), (46). Используем функцию

$$
p_{n}=\frac{1}{\dot{u}_{n}}\left(e^{w_{n}}+1\right)\left(e^{w_{n-1}}+1\right),
$$

которая является плотностью локального закона сохранения уравнения $(2): D_{t} p_{n}=$ $-(T-1) e^{w_{n-1}}$. Нужные нам формулы имеют вид

$$
\hat{p}_{n}^{(1)}=\ln p_{n}+\ln \phi\left(w_{n}\right), \quad \hat{p}_{n}^{(2)}=2 p_{n}+(T-1)\left(p_{n}\left(2-\phi\left(w_{n}\right)\right)\right) .
$$


Заметим, что если плотность закона сохранения умножить на число и прибавить к ней тривиальную плотность вида (49), то закон сохранения существенно не изменится. Так связаны $\hat{p}_{n}^{(1)}$ и $p_{n}^{(1)}$ :

$$
\hat{p}_{n}^{(1)}=-p_{n}^{(1)}+(T-1)\left(u_{n}+u_{n-1}+\ln \phi\left(w_{n-1}\right)\right) .
$$

Таким образом, проверив четыре условия интегрируемости, мы получили три существенно разных и нетривиальных закона сохранения с плотностями $\dot{u}_{n}$ и $p_{n}^{(1)}, p_{n}$ из (50), (51).

\section{5. ЗАКЛЮЧЕНИЕ}

Мы вывели условия интегрируемости для уравнений вида (1), которые являются небходимыми условиями существования высших симметрий. Стандартная схема симметрийного метода, которая сводится к исследованию приближенных решений $L_{n}$ вида (17) уравнения (13), не дает в этом случае приемлемых результатов: возникающие условия интегрируемости невозможно использовать. С трудностью удается справиться при помощи леммы о "тривиальном" точном решении $\Lambda_{n}$ уравнения (13). Лемма позволяет построить вместо $L_{n}$ приближенные решения $L_{n}^{-}$и $L_{n}^{+}$ уравнения (13) по формулам (39), (40). Теперь, вычисляя коэффициенты этих решений и применяя формулу (20), мы получаем из $L_{n}^{-}$стандартные условия интегрируемости (41), (43), которые легко использовать, а из решения $L_{n}^{+}-$нестандартные условия (45), (46).

Условия (41), (43) аналогичны тем, которые возникали прежде в работах [7] при исследовании аналогов цепочек Тоды и Вольтерра. Их нетрудно проверить для любого заданного уравнения (1), используя эквивалентность соотношений (48) и (49). Таким образом, эти условия дают удобный инструмент для проверки уравнений вида (1) на интегрируемость. Если уравнение удовлетворяет всем трем условиям интегрируемости, мы получаем для него три локальных закона сохранения. Условия (41), (43) можно также использовать для поиска новых интегрируемых примеров, исследуя уравнения вида (1) с небольшим функциональным произволом, или даже для полной классификации интегрируемых уравнений из этого класса.

Следует заметить, что аналогичным образом, продолжая вычисление коэффициентов решения $L_{n}^{-}$и применяя формулу (20), можно проводить дополнительную проверку заданного уравнения на интегрируемость, получать для него дополнительные законы сохранения, а также выписать еще одно-два условия интегрируемости в самом общем случае.

Условия интегрируемости (45), (46) принципиально отличаются от условий (41), (43). Они имеют вид локальных законов сохранения с неизвестной плотностью и при исследовании классов цепочек Тоды и Вольтерра не встречались. Неясно, как использовать эти условия для проверки заданного уравнения на интегрируемость и для классификации. Научиться применять такие условия интегрируемости - это нерешенная задача.

Точное решение $\Lambda_{n}$ уравнения (13), которое обсуждается в лемме и является квадратным корнем из оператора умножения на единичную матрицу, в литературе 
по симметрийному методу встречается впервые. Имеются и другие случаи, когда существует подобное точное решение, помогающее преодолеть аналогичные теоретические трудности. Это, во первых, класс дискретно-дифференциальных систем вида

$$
\dot{u}_{n}=f\left(u_{n+1}, u_{n}, v_{n}\right), \quad \dot{v}_{n}=g\left(v_{n-1}, v_{n}, u_{n}\right),
$$

содержащий гамильтоновую форму записи известных интегрируемых релятивистских цепочек вида (1), включая релятивистскую цепочку Тоды (2) (см. работы [6], [9], [10]). Другой класс состоит из систем уравнений с частными производными

$$
u_{t}=u_{x x}+f\left(u, v, u_{x}, v_{x}\right), \quad v_{t}=-v_{x x}+g\left(u, v, u_{x}, v_{x}\right),
$$

к которым относится расщепленное нелинейное уравнение Шредингера или система Абловица-Каупа-Ньюэлла-Сегура (о применении симметрийного метода к таким системам см., например, работы [5], [11]). Технический прием, связанный с подобным решением $\Lambda_{n}$, может оказаться полезным и в других задачах.

Благодарности. Работа поддержана РФФИ (гранты № 04-01-00190, 06-0192051-KЭ_a).

\section{Список литературы}

[1] S. N. M. Ruijsenaars, Comm. Math. Phys., 133 (1990), 217-247.

[2] Yu. B. Suris, J. Phys. A, 30 (1997), 1745-1761.

[3] В.Э. Адлер, А. Б. Шабат, ТМФ, 111 (1997), 323-334.

[4] V. V. Sokolov, A. B. Shabat, Sov. Sci. Rev. Sect. C, 4 (1984), 221-280; A. V. Mikhailov, A. B. Shabat, V. V. Sokolov, "The symmetry approach to classification of integrable equations", What is Integrability? ed. V. E. Zakharov, Springer, Berlin, 1991, 115-184.

[5] А. В. Михайлов, А. Б. Шабат, Р. И. Ямилов, УМН, 42:4 (1987), 3-53.

[6] В. Э. Адлер, А. Б. Шабат, Р. И. Ямилов, ТМФ, 125 (2000), 355-424.

[7] Р. И. Ямилов, УМН, 38:6 (1983), 155-156; R. I. Yamilov, "Classification of Toda type scalar lattices", Nonlinear evolution equations and dynamical systems NEEDS'92, Proc. of the Eighth International Workshop (Dubna, Russia, 1992), eds. V. Makhankov, I. Puzynin, O. Pashaev, World Scientific, River Edge, NJ, 1993, 423-431.

[8] D. Levi, R. Yamilov, J. Math. Phys., 38 (1997), 6648-6674; R. Yamilov, D. Levi, J. Nonlinear Math. Phys., 11 (2004), 75-101.

[9] R. Yamilov, J. Phys. A, 39 (2006), R541-R623.

[10] Р. И. Ямилов, ТМФ, 139 (2004), 209-224.

[11] A. V. Mikhailov, A. B. Shabat, R. I. Yamilov, Comm. Math. Phys., 115 (1988), 1-19. 